\title{
THREE-YEAR FOLLOWUP OF PATIENTS TREATED WITH LOWER ENERGY MICROWAVE THERMOTHERAPY
}

\author{
M. J. A. M. DE WILDT, F. C. H. D'ANCONA, M. HUBREGTSE, S. ST. C. CARTER, F. M. J. DEBRUYNE \\ AND J. J. M. C. H. DE LA ROSETTE \\ From the Departments of Urology, Nijmegen University Hospital, Nijmegen, The Netherlands and Hammersmith Hospital Trust, London, \\ United Kingdom
}

\section{ABSTRACT}

Purpose: A retrospective study was done to investigate the long-term outcome of patients treated with lower energy transurethral microwave thermotherapy.

Materials and Methods: A total of 305 patients with lower urinary tract symptoms and benign prostatic hypertrophy underwent transurethral microwave thermotherapy according to a similar protocol at 2 centers.

Results: After 3 years of followup 133 patients who had undergone transurethral microwave thermotherapy alone were available for study. During this observation period significant symptomatic improvement from baseline and an improved maximum flow rate of $2.6 \mathrm{ml}$. per second were noted. Of the patients 125 underwent invasive or medical treatment.

Conclusions: After 3 years of followup lower energy transurethral microwave thermotherapy showed significant and durable improvements in baseline parameters in $52 \%$ of the patients.

KEY WORDS: prostate; prostatic hypertrophy; microwaves; hyperthermia, induced

During the last decade many different alternatives to surgical resection of the prostate in patients with lower urinary tract symptoms and benign prostatic hyperplasia (BPH) have been introduced, including use of different oral medications, such as $5 \alpha$-reductase inhibitors and $\alpha 1$-adrenergic antagonists, as well as several minimally invasive approaches. ${ }^{1,2}$ Although surgical resection effectively relieves bladder outlet obstruction with a consequent decrease in symptoms, the popularity of these alternative therapeutic options in the treatment of BPH is based on the potential decrease in morbidity and costs. The use of heat applied by different generators (for example ultrasound, radiofrequency, laser and microwave devices), appears to be the most promising alternative. ${ }^{3-6}$ Transurethral microwave thermotherapy offers great potential as an outpatient anesthesia-free 1-session procedure. Presently, of the many different thermotherapy devices the Prostatron* unit has received the most attention and has been investigated extensively with more than 25,000 treatments performed worldwide. Several studies of this device report substantial and significant subjective improvement. An overall improvement rate of approximately $70 \%$ in the Madsen-Iversen symptom score ${ }^{7}$ compared to baseline usually is noted. Improvement in urinary performance also is encouraging, with improvement in maximum flow rate of approximately 2 to $3 \mathrm{ml}$. per second (approximately $35 \%$ ) from baseline and a similar decrease in post-void residual of approximately $35 \% .6$ The mechanism of action may be related to thermal damage of prostate tissue and not to the effect of urethral manipulation as shown by several randomized studies of transurethral microwave thermotherapy versus sham therapy. ${ }^{8,9}$ More importantly, Dahlstrand et al showed that the symptomatic improvement that occurred after transurethral microwave thermotherapy was statistically identical to that noted after transurethral resection of the prostate. ${ }^{10}$ However, the effect of transurethral microwave thermotherapy on uroflowmetry parameters was less pronounced compared to that of transurethral re-

Accepted for publication June 7, 1996.

* Technomed, Lyon, France. section. Subjective and objective parameters remained stable for 3 years of observation. ${ }^{6}$

We have gained a large experience with the Prostatron device. Since the end of 1990 treatments with the lower energy protocol (Prostasoft version 2.0) have been performed. We conducted a retrospective study of patients treated with this protocol at 2 different centers and report the long-term results.

\section{MATERIAL AND METHODS}

From December 1990 to December 1992, 305 men with lower urinary tract symptoms and BPH were treated with the Prostatron device. Pretreatment assessment included patient history with Madsen-Iversen symptom scores, ${ }^{7}$ physical examination with digital rectal examination, urinalysis, urine culture, transrectal ultrasound of the prostate with calculation of prostate volume using the formula of Terris and Stamey, ${ }^{11}$ and uroflowmetry with measurement of postvoid residual by abdominal ultrasound of the bladder using the ellipsoid technique.

The majority of patients were included in a range of prospective trials conducted according to more or less similar protocols. Criteria to enter the study were patient age older than $\mathbf{4 5}$ years, lower urinary tract symptoms longer than 3 months in duration, a Madsen-Iversen symptom score of 8 or more, urinary maximum flow of $15 \mathrm{ml}$. per second or less and post-void residual of $350 \mathrm{ml}$. or less. Exclusion criteria were urinary retention, prostate carcinoma, acute or chronic prostatitis, urethral stricture, intravesical pathological condition (stones or neoplasm), neurogenic bladder dysfunction, urinary tract infection, isolated enlargement of the middle lobe, use of drugs influencing bladder or prostate function, disorders of blood flow or coagulation and diabetes mellitus.

Transurethral microwave thermotherapy has been described previously. ${ }^{12}$ After treatment patients were asked to remain in the department until satisfactory voiding had been established. In case of retention a urethral catheter was placed, usually for 1 week. All patients were treated at least 3 years ago. Data on symptom scores and uroflowmetry results were analyzed retrospectively at baseline, and 12,24 1959 
and 36 months after treatment. Maximum flow was corrected for artifacts by 2 independent observers using the 2 -second method. ${ }^{13}$ Voided volume was correlated with post-void residual to provide a voiding fraction using the formula: percent voiding fraction $=$ voided volume $/($ voided volume + post void residual). ${ }^{14}$

In case of further surgical or medical intervention the date and type of treatment were noted. When a patient did not return for further followup and there was no outcome record in the file a detailed questionnaire including the MadsenIverson symptom score was mailed to the patient. If the Madsen-Iverson symptom score was missing at the 3-year visit to the outpatient clinic a score was obtained via telephone interview. A patient was considered lost to followup if there were no data after the last visit to the outpatient clinic despite several attempts to contact him via mail or telephone. If a patient was not satisfied with the result of transurethral microwave thermotherapy and, therefore, he took medications for the lower urinary tract symptoms ( $5 \alpha$-reductase, an $\alpha$ blocker or anticholinergics) or underwent surgery the uroflowmetry or symptom score data were not considered when evaluating followup results. Finally, posttreatment morbidity was noted if the patient experienced urinary incontinence, urethral stricture or other treatment related complaints.

\section{RESULTS}

Of the 305 patients 73 were treated in London and 232 in Nijmegen. The former patients on average had smaller prostates, voided with larger volumes, had a larger post-void residual and, consequently, had a smaller voiding fraction. All other parameters used in the study were equally distributed and statistically similar (table 1). For the total group mean age at baseline plus or minus standard deviation was $65.3 \pm 7.3$ years (range 45 to 87 ) and average prostate volume was $48.6 \pm 20.7 \mathrm{~cm}^{3}$ (range 15 to 133 ). After 3 years of followup 133 patients, who had no additional medical or surgical treatment, were available for study (table 2). Of 140 letters mailed to patients 99 were returned and 41 men were considered lost to followup. If no data were available at 1 and/or 2 years and the patient returned for further followup after 3 years or when he completed the mailed questionnaire he was considered to have had a missed visit at 1 and/or 2 years of followup.

Table 3 shows the paired data for improvement in the main indexes from baseline. After 1 year there was a $56 \%$ decrease in symptom score that gradually decreased further to 53 and $34 \%$ at 2 and 3 years of followup, respectively. Nevertheless, symptoms remained statistically improved over baseline. Improvement in maximum flow remained stable at 24, 20 and $27 \%$ of cases after 1,2 and 3 years of followup, respectively. Improvement in post-void residual and voiding fraction also remained constant for the 3 years of observation, although improvement in voided percentage over baseline was no longer statistically significant at 3 years. This finding may be due to the small number of patients whose uroflowmetry data were available at this visit.

TABLE 1. Baseline characteristics for the 2 centers

\begin{tabular}{lccc}
\hline & \multicolumn{2}{c}{ Mean \pm SD } & \\
\cline { 2 - 3 } & $\begin{array}{c}\text { Nijmegen } \\
(232 \text { pts. })\end{array}$ & $\begin{array}{c}\text { London } \\
(73 \mathrm{pts} .)\end{array}$ & \\
& $64.9 \pm 7.2$ & $66.8 \pm 7.5$ & \\
\hline Pt. age (yrs.) & $49.9 \pm 19.7$ & $43.6 \pm 23.4$ & 0.051 \\
Prostate vol. (cm. ${ }^{3}$ ) & $12.7 \pm 3.5$ & $13.5 \pm 3.4$ & $0.031^{*}$ \\
Madsen-Iverson symptom score & & & \\
Uroflowmetry: & $9.0 \pm 2.8$ & $9.4 \pm 2.7$ & 0.375 \\
Max. flow rate (ml./sec.) & $223 \pm 113$ & $258 \pm 121$ & $0.045^{*}$ \\
Voided vol. (ml.) & $65 \pm 68$ & $139 \pm 98$ & $<0.001^{*}$ \\
Post-void residual (ml.) & $79 \pm 18$ & $68 \pm 19$ & $<0.001^{*}$ \\
\% Voiding fraction & * Significant difference, Student's t test, $\alpha=0.05$. &
\end{tabular}

TABLE 2. Number of patients, additional treatments and losses to followup

\begin{tabular}{lrrrrr} 
& \multicolumn{3}{c}{ No. Pts. } & \\
\cline { 2 - 5 } & & 1 & 2 & 3 & Total No. Pts. \\
& Yr. & Yrs. Yrs. & \\
\hline Transurethral microwave thermotherapy only & 233 & 112 & 133 & 133 \\
Medication & 18 & 11 & 16 & 45 \\
Invasive procedure after prior use of medica- & 2 & 9 & 5 & 16 \\
$\quad$ tion & 22 & 23 & 19 & 64 \\
Invasive procedure & 1 & 2 & 3 & 6 \\
Death & 17 & 19 & 5 & 41 \\
Lost to followup & 12 & 69 & - & - \\
Missed visit & At baseline 305 patients underwent transurethral microwave thermother- \\
apy alone. & & & &
\end{tabular}

Stratification of the data for patients treated with transurethral microwave thermotherapy alone by the percentage change in outcome at 1,2 and 3 years of followup as a definition of success as suggested by Food and Drug Administration guidelines is shown in table 4. The proportion of patients with $50 \%$ or more symptomatic improvement remained stable during the first 2 years and decreased to $39 \%$ at 3 years of followup. The proportion of patients with $50 \%$ or more improvement in maximum flow remained durable at 26,23 and $27 \%$ at 1,2 and 3 years of followup, respectively. Except for the greater symptomatic improvement at 1 year for the London patients, there appeared to be no statistically significant difference between the London and Nijmegen groups in amount of improvement in any main index (table 5 ). At 3 years of followup 133 of 258 patients (52\%) with available data had undergone transurethral microwave thermotherapy alone. Overall, 80 of 219 men (31\%) underwent an invasive procedure, including a second transurethral microwave thermotherapy in 8 , transurethral resection of the prostate in 45 , incision of the prostate in 3 , laser prostatectomy in 17 , suprapubic prostatectomy in 5 and radical prostatectomy after diagnosis of prostate cancer in 1.

Of the 258 patients $60(23 \%)$ were not satisfied with the result of transurethral microwave thermotherapy and they began medical therapy ( 42 with $\alpha$ blockers, 5 with $5 \alpha$ reductase and 12 with anticholinergic drugs). One patient began flutamide therapy when prostate cancer was diagnosed. Of these men 16 were not content with medical therapy and they finally underwent surgical intervention. The figure illustrates the Kaplan-Meier plot of early termination of the study due to starting medication and undergoing an invasive procedure. Patients waited an average of $1.4 \pm 0.8$ years (range 0.5 to 2.9 ) before initiating medical therapy and $1.5 \pm 0.8$ years (range 0.25 to 2.9 ) before undergoing surgery due to dissatisfaction with the result after transurethral microwave thermotherapy.

No statistically significant difference in any baseline parameter was found at 3 years after transurethral microwave thermotherapy between patients who had a $50 \%$ or greater response in symptom score or maximum flow and those who underwent subsequent medical or surgical treatment. After treatment 82 of 305 patients $(27 \%)$ required a transurethral catheter due to urinary retention, which was removed 1 week after treatment in the majority of patients. Three of 258 patients $(1.2 \%)$ had recurrent urinary tract infections requiring antibiotics, $8(3.1 \%)$ had prolonged macroscopic hematuria, $1(0.4 \%)$ had a urethral stricture and $4(1.6 \%)$ had urge incontinence. Finally, 4 patients were diagnosed with prostate cancer by prostate biopsies (2) and histology of resected tissue after transurethral resection of the prostate (2).

\section{DISCUSSION}

The aim of any treatment for lower urinary tract symptoms and $\mathrm{BPH}$ is to achieve symptomatic relief with a correspond- 
TABLE 3. Followup paired data for baseline parameters in patients undergoing transurethral microwave thermotherapy without additional treatment

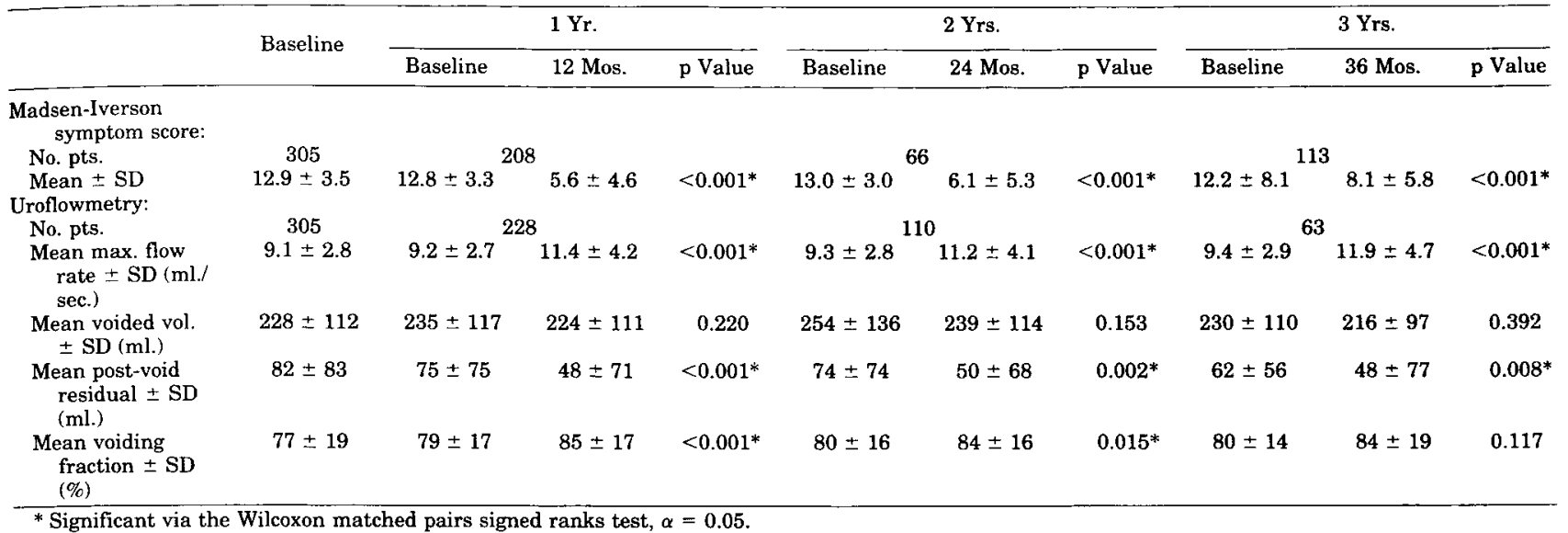

TABLE 4. Response rates of main indexes after 1, 2 and 3 years in patients undergoing transurethral microwave thermotherapy alone shown as percent of patients with available data

\begin{tabular}{lcccc}
\hline & \multicolumn{4}{c}{$\begin{array}{c}\text { Improvement From Baseline } \\
\text { Parameter }\end{array}$} \\
\cline { 2 - 5 } & $\begin{array}{c}\text { Less } \\
\text { Than 25 }\end{array}$ & $25-50$ & $\begin{array}{c}\text { More } \\
\text { Than 50 }\end{array}$ & $\begin{array}{c}\text { More } \\
\text { Than 75 }\end{array}$ \\
\hline Madsen-Iverson symptom score at yr.: & 19 & 21 & 60 & 34 \\
1 & 23 & 15 & 62 & 26 \\
2 & 48 & 13 & 39 & 27 \\
3 & & & & \\
Max. flow rate (ml./sec.) at yr.: & 54 & 20 & 26 & 15 \\
1 & 57 & 20 & 23 & 14 \\
2 & 51 & 22 & 27 & 18 \\
3 & & &
\end{tabular}

TABLE 5. Difference in improvement in baseline parameters

\begin{tabular}{llll} 
& \multicolumn{2}{c}{ Mean \pm SD } & \multirow{2}{*}{ p Value } \\
\cline { 2 - 3 } & London & Nijmegen & \\
\hline Madsen-Iversen symptom score at yr: & & & \\
1 & $9.2 \pm 4.6$ & $6.5 \pm 4.9$ & $0.001^{*}$ \\
2 & $8.5 \pm 5.8$ & $5.8 \pm 5.7$ & 0.065 \\
3 & $5.9 \pm 6.2$ & $3.8 \pm 5.9$ & 0.210 \\
Max. flow rate (ml./sec.) at yr.: & & & \\
1 & $2.7 \pm 3.8$ & $2.0 \pm 3.7$ & 0.249 \\
2 & $1.6 \pm 3.8$ & $1.9 \pm \mathbf{4 . 3}$ & 0.701 \\
3 & $1.7 \pm 7.2$ & $2.6 \pm 4.0$ & 0.560 \\
\hline * Significant via Student's t test for independent samples, $\alpha=\mathbf{0 . 0 5 .}$
\end{tabular}

ing decrease in bladder outlet obstruction. Symptomatic improvement is determined by a decrease in symptom scores. Traditionally, symptomatic efficacy of transurethral microwave thermotherapy is evaluated with the Madsen-Iverson symptom score, which was the only commonly used symptom score at the time of the initial trials in 1991. Decreased bladder outlet obstruction is usually evaluated by increased urinary flow rate since urodynamic pressure-flow studies are not usually applied. A treatment outcome can be expressed as mean changes or a percentage improvement in the parameters. Short-term results with these methods of evaluation and morbidity of transurethral microwave thermotherapy have been extensively reported. ${ }^{6}$ However, data on the durability of this treatment and re-treatment rate are limited to only a few reports. $9,15,16$

Our 56\% improvement rate at 1 year of followup is lower than the approximately $70 \%$ improvement rate reported in the literature. The same finding accounts for the objective maximum flow improvement of $2.2 \mathrm{ml}$. per second $(24 \%)$,

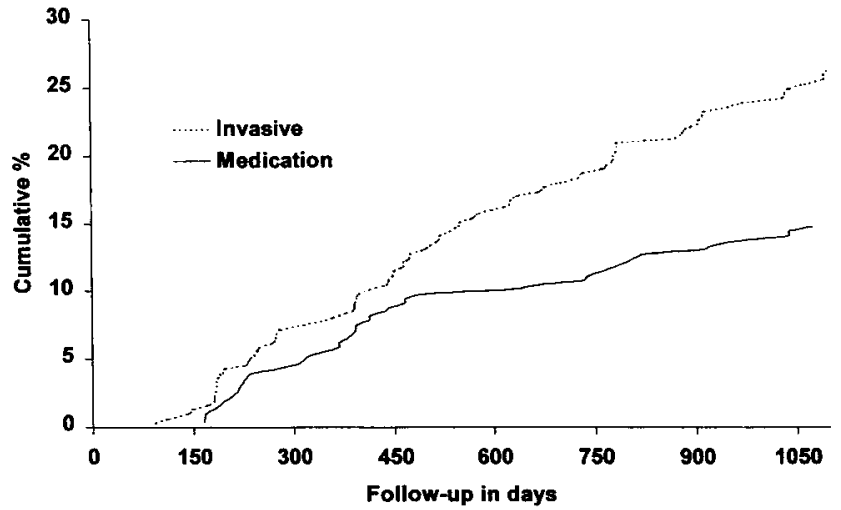

Kaplan-Meier plot presenting early termination of study. Each individual line represents cumulative percentage of dropouts of patients.

which also is slightly less compared to the 3 to $4 \mathrm{ml}$. per second (approximately 35\%) improvement in maximum flow reported in the literature. Finally, the $36 \%$ decrease in postvoid residual seems to be more comparable, although data were limited and reports varied from 22 to $69 \%$ improvement. ${ }^{6}$ On the other hand, the percentage improvement from baseline in main outcome indexes using the Food and Drug Administration stratification guidelines was similar to that of prior studies. Our study showed $50 \%$ or greater improvement in symptom scores and maximum flow rate in 60 and $26 \%$ of patients, respectively. Data from the literature revealed $50 \%$ or greater improvement in symptoms and maximum flow rate in 62 and $36 \%$ of patients, respectively. ${ }^{8}$ Furthermore, our study showed that the achieved improvement in symptoms and urinary performance remained durable and more or less stable for a 3-year observation period, which is in accordance with others who reported 2 and 3-year followup data in a randomized study of transurethral microwave thermotherapy versus transurethral resection of the prostate.6,9 Nevertheless, there appeared to be a trend toward deterioration in symptoms with duration of followup, and that at 3 years the mean Madsen-Iversen symptom score was 8.1 , which was just above the entry level of our study, particularly when one considers that patients without additional treatment at 3 years of followup represent the best responders.

Where should transurethral microwave thermotherapy be placed among all available treatment options for patients 
with lower urinary tract symptoms and BPH? The objective improvement after lower energy transurethral microwave thermotherapy is not comparable to what is achieved after surgical resection of the prostate as shown by Dahlstrand et al. 9 The magnitude of improvement in urinary performance seems to be more in the ranges achieved with medical therapy. In an open extension North American study Stoner reported significant improvement in maximum flow from baseline values of $2.4 \mathrm{ml}$. per second in 156 patients 36 months after beginning finasteride treatment. ${ }^{1}$ Lepor reported similar changes in 103 patients treated with terazosin after 3 years of followup. ${ }^{2}$ Improvement in maximum flow ranged from 2.3 to $4.0 \mathrm{ml}$. per second greater than the baseline value at 3 to 42 months of followup, with $30 \%$ or more improvement in flow in 40 to $59 \%$ of patients. However, patients still using medication at 3 years of followup should also be considered the best responders, which inherently skew the data. Tables 3 and 4 show similar improvements at 3 years after transurethral microwave thermotherapy with a $2.5 \mathrm{ml}$. per second improvement in flow and $25 \%$ or greater improvement in $49 \%$ of patients. Also, symptomatic improvements with the alternative treatment options seem to be more or less comparable. Since the studies reporting on longterm followup use different symptom scores to evaluate treatment outcome, the only way to compare these studies is by using the relative or percentage improvement. Lepor documented a $30 \%$ or greater improvement in symptom score in 62 to $77 \%$ of patients at 3 to 42 months of observation. ${ }^{2}$ Stoner reported a mean decrease in symptom score of 3.6 points after 36 months in the extended study of finasteride. ${ }^{1}$ However, although improvement was significant compared to baseline values and to the placebo control group, it only accounted for approximately $18 \%$ improvement overall. Our study of transurethral microwave thermotherapy showed that symptomatic improvement after 3 years was 4.1 points, which accounted for $34 \%$ improvement over baseline. Furthermore, a $25 \%$ or greater improvement was achieved in $52 \%$ of patients (table 3 ).

Another point that must be addressed in evaluation of transurethral microwave thermotherapy is the repeat treatment rate. Every treatment option for lower urinary tract symptoms and BPH has a failure rate. In the case of initial surgical treatment, further surgery is performed because of complications (urethral stricture or bladder neck sclerosis) or recurrent disease. Table 2 shows that repeat invasive treatment after initial transurethral microwave thermotherapy was necessary in 24 of $287(8 \%), 32$ of 224 (14\%) and 24 of 173 (14\%) of patients with available data at 1,2 and 3 years of followup, respectively, which accounts for a total of 80 of 258 $(31 \%)$ requiring re-treatment in 3 years. The United States Prostatron transurethral microwave thermotherapy group recently presented the long-term results of a Food and Drug Administration study. ${ }^{17}$ In contradistinction to our study, they reported a significantly lower re-treatment rate of $11 \%$ after transurethral resection of the prostate. However, $29 \%$ of the men reported to have changed to some form of medical treatment. These differences are likely to be explained by difference in department policy. In our study many patients were treated with laser prostatectomy due to several protocols that were conducted with this modality at that time, whereas in the United States it appeared that medication was the first choice of treatment when patients did not experience improvement after transurethral microwave thermotherapy. Nevertheless, the United States and our studies noted a comparable and significant improvement in 52 and $51 \%$ of patients at 3 and 4 years of followup, respectively. Available data in the literature on re-treatment rates after surgical resection of the prostate have some flaws, since the reported 1.8 to $15.5 \%$ re-treatment rate mainly depended on the observation period. 18 The largest documented retrospective study is that of Roos et al, who reported on 50,000 patients undergoing transurethral resection of the prostate between 1963 and $1985 .^{19}$ The re-treatment rate for a second prostatectomy was 2.3 to $4.3,8.9$ to 9.7 and 12.0 to $15.5 \%$ after 1,5 and 8 years, respectively. In this respect the United States long-term results appear to be comparable and, since none of the transurethral prostatectomy studies reported re-treatment with medication, the overall re-treatment rates after transurethral microwave thermotherapy and transurethral resection of the prostate might actually be similar. Unfortunately, neither the finasteride nor terazosin studies reported on the fate of patients whose treatment was considered to have failed.

Our re-treatment rate in patients treated with transurethral microwave thermotherapy was greater than that after transurethral resection of the prostate. However, a price must be paid in terms of morbidity. In this respect transurethral microwave thermotherapy seems to be favorable. We only reported minor complications after transurethral microwave thermotherapy except for the $27 \%$ retention rate, which is much greater than the reported $6.5 \%$ rate noted after surgical intervention. ${ }^{20}$ Since transurethral microwave thermotherapy is an invasive procedure a urethral stricture rate of $0.4 \%$ ( 1 of 258 cases) can be considered slight compared to the 2 to $20 \%$ incidence after surgery. ${ }^{21}$ No patient reported stress incontinence although $4(1.6 \%)$ experienced urge incontinence after transurethral microwave thermotherapy, possibly due to detrusor instability. Finally, the incidence of prostate cancer in only 4 of 258 patients ( $1.6 \%$ ) appears to be low compared to available data on prostatectomy studies, which may be explained by the fact that patients were screened before entry into the study and that only 50 of 258 (19\%) actually had available histological data from resected tissue.

Like several other clinical reports of transurethral microwave thermotherapy, our study showed a great interindividual difference in treatment outcome regarding objective and subjective parameters, which has led many investigators to search for selection criteria that could predict clinical outcome. A multicenter study of responders versus nonresponders to transurethral microwave thermotherapy showed that none of the baseline clinical parameters could predict treatment outcome. ${ }^{22}$ Our study supports this conclusion, since no difference in the main treatment indexes was found between patients who could be considered to have a good response at 3 years of followup and those in whom treatment actually failed. To date the only predictive factor for treatment outcome can be obtained from urodynamic studies with pressure-flow analysis as demonstrated by Tubaro et al in a European multicenter study. ${ }^{22}$ Patients with constrictive urodynamic obstruction showed favorable improvement in symptoms and voiding parameters over those with predominantly compressive obstruction. ${ }^{22}$

Furthermore, thermometry studies have shown that not all prostates reach the maximum temperature intended, because the thermoregulation of tissue in every patient differs significantly. ${ }^{23}$ In addition, the correlation between the achieved intraprostatic temperature and treatment outcome suggests that the greater the intraprostatic temperature, the better the clinical results. ${ }^{24}$ This finding has led to modification of the treatment software by increasing rectal threshold temperature and energy levels, resulting in fewer interruptions during treatment and a mean $40 \%$ increase in total energy delivered to the prostate. ${ }^{25}$ Initial clinical experience in phase II studies with high energy transurethral microwave thermotherapy using Prostasoft version 2.5 has shown increased objective improvement with comparable symptomatic improvement. ${ }^{25,26}$ The relief of urodynamic bladder outlet obstruction and a cavity on transrectal ultrasound of the prostate 3 months after high energy transurethral microwave thermotherapy might also indicate improved efficacy and durability in the long term. 
In conclusion, lower energy transurethral microwave thermotherapy results in significant symptomatic improvement in $52 \%$ of patients, while objective improvement is approximately $3 \mathrm{ml}$. per second at 3 years of followup. The shortterm and long-term morbidity is acceptably low.

\section{REFERENCES}

1. Stoner, E.: Three-year safety and efficacy data on the use of finasteride in the treatment of benign prostatic hyperplasia. Urology, 43: 284, 1994.

2. Lepor, H.: Long-term efficacy and safety of terazosin in patients with benign prostatic hyperplasia. The Terazosin Research Group. Urology, 45: 406, 1995.

3. Madersbacher, S., Kratzik, C., Suzani, M. and Marberger, M.: Tissue ablation in benign prostatic hyperplasia with highintensity focused ultrasound. J. Urol., 152: 1956, 1994.

4. Schulman, C. C. and Zlotta, A. R.: Transurethral needle ablation of the prostate for treatment of benign prostatic hyperplasia: Early clinical experience. Urology, 45: 28, 1995.

5. Cowles, R. S., III, Kabalin, J. N., Childs, S., Lepor, H., Dixon, C., Stem, B. and Zabbo, A.: A prospective randomized comparison of transurethral resection to visual laser ablation of the prostate for the treatment of benign prostatic hyperplasia. Urology, 46: 155, 1995.

6. de Wildt, M. J. A. M. and de la Rosette, J. J. M. C. H.: Transurethral microwave thermotherapy: an evolving technology in the treatment of benign prostatic enlargement. Brit. J. Urol., 76: $531,1995$.

7. Ogden, C. W., Reddy, P., Johnson, H., Ramsay, J. W. A. and Carter, S. St. C.: Sham versus transurethral microwave thermotherapy in patients with symptoms of benign prostatic bladder outflow obstruction. Lancet, 341: 14, 1993.

8. Madsen, $O$. M. and Iversen, P.: A point system for selecting operative candidates. In: Benign Prostatic Hyperthrophy. Edited by F. Hinman. New York: Springer-Verlag, chapt. 79, pp. $763-765,1983$.

9. de Wildt, M. J. A. M., Hubregtse, M., Ogden, C. W., Debruyne, F. M. J., Carter, S. St. C. and de la Rosette, J. J. M. C. H.: A 12 month study of the placebo effect in TUMT. Brit. J. Urol., 77: $221,1996$.

10. Dahlstrand, C., Waldén, M., Geirsson, G. and Pettersson, S.: Transurethral microwave thermotherapy versus transurethral resection for symptomatic benign prostatic obstruction: a prospective randomized study with a 2-year follow-up. Brit. J. Urol., 76: 614, 1995 .

11. Terris, M. K. and Stamey, T. A.: Determination of prostate volume by transrectal ultrasound. J. Urol., 145: 984, 1991.

12. Carter, S. St. C., Patel, A., Reddy, P., Royer, P. and Ramsay, J. W. A.: Single-session transurethral microwave thermotherapy for the treatment of benign prostatic obstruction. J. Endourol., 5: 137, 1991.
13. Grino, P. B., Bruskewitz, R., Blaivas, J. G., Siroky, M. B., Anderson, J. T., Cook, T. and Stoner, E.: Maximum urinary flow rate by uroflowmetry: automated or visual interpretation. J. Urol., 149: 339, 1993.

14. Cucchi, A.: Urinary flow rate in benign prostatic hyperthrophy in relation to the degree of obstruction of the vesical outlet. Brit. J. Urol., 69: 272, 1992.

15. Ersev, D., Ilker, Y. and Kuyumcuoğlu, U.: Two years of follow-up in 112 patients treated by trans-urethral microwave thermotherapy. Read at XIth Congress of the European Urological Association of Urology. Berlin, Germany, p. 643A, July 13-16, 1994.

16. Homma, Y. and Aso, Y.: Transurethral microwave thermotherapy for benign prostatic hyperplasia: a 2-year follow-up study. J. Endourol., 7: 261, 1993.

17. Blute, M., Hanson, K., Lynch, J., Tomera, K., Cope, P., Sankey, N., Wernecke, C., McKiel, C. and Carter, S. St.C.: United States Prostatron TUMT Study-4 year followup and quality of life. J. Urol., part 2, 155: 403A, abstract 370, 1996.

18. de Wildt, M. J. A. M., de la Rosette, J. J. M. C. H. and Debruyne, F. M. J.: Retreatment rate. In: Benign Prostatic Hyperplasia. Recent Progress in Clinical Research and Practice. Edited by K. Kurth and D. W. W. Newling, New York: Wiley-Liss, Inc., pp. 597-613, 1994.

19. Roos, N. P., Wennberg, J. E., Malenka, D. J., Fisher, E. S., McPherson, K., Andersen, T. F., Cohen, M. M. and Ramsey, E.: Mortality and reoperation after open and transurethral resection of the prostate for benign prostatic hyperplasia. New Engl. J. Med., 320: 1120, 1989.

20. de Wildt, M. J. A. M., Tubaro, A., Höfner, K., Carter, S. St. C., de la Rosette, J. J. M. C. H. and Devonec, M.: Responders versus nonresponders to transurethral microwave thermotherapy: a multicenter retrospective analysis. J. Urol., 154: 1775, 1995.

21. Tubaro, A., Carter, S. St. C., de la Rosette, J. J. M. C. H., Höffner, K., Trucchi, A., Ogden, C., Miano, L., Valenti, M., Jonas, U. and Debruyne, F.: The prediction of clinical outcome from transurethral microwave thermotherapy by pressureflow analysis: a European multicenter study. J. Urol., 153: 1526, 1995.

22. Devonec, M., Berger, N., Fendler, J. P., Joubert, P., Nasser, M. and Perrin, P.: Thermoregulation during transurethral microwave thermotherapy: experimental and clinical fundamentals. Eur. Urol., suppl. 1, 23: 63, 1993.

23. Carter, S. St.C. and Ogden, C. W.: Intraprostatic temperature v. clinical outcome in T.U.M.T. Is the response heat-dose dependent? J. Urol., part 2, 151: 416A, abstract, 756, 1994.

24. de la Rosette, J. J. M. C. H., Tubaro, A., Höfner, K. and Carter, S. St.C.: Transurethral microwave thermotherapy: past, present and future. World J. Urol., 12: 352, 1994.

25. Devonec, M., Carter, S. St. C., Tubaro, A., de la Rosette, J., Höfner, K., Dahlstrand, C. and Perrin, P.: Microwave therapy. Curr. Opin. Urol., 5: 3, 1995. 